\title{
Rationale and Structure for a New Center for Studies on Prevention of Alzheimer's Disease (StoP-AD)
}

\author{
J.C.S. Breitner ${ }^{1,2}$, J. Poirier1,2, P.E. Etienne ${ }^{1,2}$, J.M. Leoutsakos ${ }^{3}$ for the PREVENT-AD Research Group ${ }^{1}$

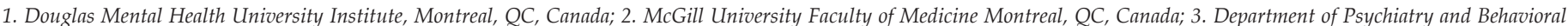 \\ Sciences, Johns Hopkins University School of Medicine, Baltimore, MD, USA
}

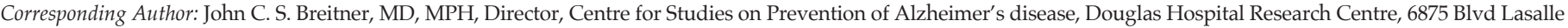
Montreal, QC H4H 1R3, Canada, john.breitner@mcgill.ca, (01)-514-761-6131, ext 3940, fax (01)-514-221-4700

\begin{abstract}
We describe events spanning over 20 years that have shaped our approach to identification of interventions that may delay symptoms in Alzheimer's disease (AD). These events motivated the development of a new Centre for Studies on Prevention of $\mathrm{AD}$ that includes an observational cohort of cognitively normal high-risk persons and INTREPAD, a nested two-year randomized placebo-controlled trial of the non-steroidal antiinflammatory drug naproxen sodium. INTREPAD enrolled 217 persons and will follow 160 in a modified intent-to-treat analysis of persons who remained on-protocol through at least one follow-up evaluation. The trial employs dual endpoints: 1) a composite global cognitive score generated by a battery of 12 psychometric tests organized into five subscales; and 2) a summary Alzheimer's Progression Score derived from latent variable modeling of multiple biomarker data from several modalities. The dual endpoints will be analyzed by consideration of their joint probability under the null hypothesis of no treatment effect, after allowing appropriately for their lack of independence.

We suggest that such an approach can be used economically to generate preliminary data regarding the efficacy of potential prevention strategies, thereby increasing the chances of finding one or more interventions that successfully prevent symptoms.
\end{abstract}

Key words: Prevention trials, biomarkers, Alzheimer's disease, pathogenesis.

\section{Introduction}

$\mathbf{W}$ e describe events leading to the development of a new Centre for Studies on Prevention of Alzheimer's Disease (AD) deriving from observations over 30 years. We review some history and key constructs that motivate our present activities. We then offer a description of our methods and the sorts of data expected over the coming two years.

\section{Historical development and rationale}

In the 1980's, studies of familial aggregation in AD led to a concept of disease risk as a monotonically increasing function of time (1-4). This notion provoked consideration to the effects of delayed onset on the lifetime incidence of AD dementia. In 1991, one of us (JCSB) suggested that a five-year delay in onset should reduce the lifetime incidence of disease by $37 \%$ (5). A year later, Zaven Khachaturian correctly noted that later onset would result not only in reduced incidence but also in decreased duration of illness, and offered an improved estimate that a five-year delay in onset would decrease the population burden of disease by $50 \%$, while a tenyear delay would reduce it by another half (6). The latter estimate was subsequently corroborated using more sophisticated methods by Brookmeyer et al. (7). Thus delay in onset became a prime strategy for prevention of symptoms.

\section{Enter APOE}

In the early 1990s we had little sense of the sorts of intervention that might achieve this sort of delay. In 1993, however, the laboratories of Drs. Allen Roses and Judes Poirier established a strong relationship between polymorphism at $A P O E$ and the risk of later-onset $\mathrm{AD}$ dementia $(8,9)$. The Roses group showed a specific association between APOE polymorphism and the distribution of $\mathrm{AD}$ onsets in a collection of families with multiple cases of AD (10). This latter report showed five- or greater-year differences in typical onset of $\mathrm{AD}$ dementia. Shortly thereafter, epidemiological studies in representative populations showed substantial APOErelated alterations in population prevalence $(11,12)$ and incidence of AD dementia $(11,13)$.

\section{Awareness of 'pre-clinical' $A D$}

Robert Katzman is often credited with the idea that Alzheimer's disease is a chronic illness with a pre-clinical (we prefer the more precise term pre-symptomatic) stage. Logically, pre-symptomatic AD should represent a window of opportunity for preventive interventions. Just as it became evident that $A P O E$ polymorphism modified the timing of $\mathrm{AD}$ symptom appearance (14), other factors might modify the incidence of $\mathrm{AD}$ dementia by affecting 
the rate at which the pre-symptomatic disease evolved, and thus the age at which symptoms emerged (15). Among strategies to accomplish this, one might attempt to simulate the effects of the less risky APOE alleles (especially $\varepsilon 2$ ) in pre-symptomatic AD.

\section{Influence of new theories on pathogenesis}

Most work in this area drew on the amyloid cascade hypothesis (16) or the potential importance of hyperphosphorylated tau proteins (17). The main focus was treatment of symptomatic disease rather than prevention, but several major prevention programs are now pursuing strategies aimed at preventing overproduction of $\mathrm{A} \beta$ peptides $(18,19)$. At least one randomized trial of an anti-tau treatment has recently been reported $(20,21)$. Some concern about these efforts (possibly excepting the API FAD trial) has arisen with recent understanding that fibrillary amyloid deposition in Late Onset AD may result more from diminished clearance than from overproduction of $\mathrm{A} \beta$ peptides (22, 23).

\section{Other strategies, including anti-inflammatory treatments}

Our group has concentrated on interventions not based on the amyloid or tau theories of AD pathogenesis, which are being pursued vigorously elsewhere. Prominent among these have been several trials of various antiinflammatory agents (24), motivated largely by epidemiological studies from the 1990's suggesting reduced incidence of $\mathrm{AD}$ dementia in aging persons exposed to anti-inflammatory treatments, primarily NSAIDs. One such trial was the Alzheimer Disease Anti-inflammatory Prevention Trial (ADAPT), a double-masked pharmaco-prevention trial of the conventional NSAID naproxen and the selective COX-2 inhibitor celecoxib (25). An initial report from ADAPT described its results as null (26). However, that report described an analysis which excluded several persons with prevalent dementia (undetected by the trial's screening procedures), and even this analysis suggested a worrisome increase in the incidence of $\mathrm{AD}$ dementia with both treatments in the first $2-3$ years following randomization. Later analyses (27) suggested that ADAPT participants exposed to naproxen developed no new cases of dementia in the $2-3$ years thereafter (in contrast to celecoxib- or placebo-assigned persons), and a subsequent growth mixture modeling analysis suggested similar results (28). Perhaps more importantly, CSF from almost 200 lumbar punctures (LPs) obtained between 21 and 41 months following the termination of treatments showed a substantially lower ratio in the CSF of total tau (t-tau) / A $\beta 1-42$ concentrations - a widely recognized marker of disease progression - in participants originally assigned to naproxen (27). Predictably, however, still later analyses of the ADAPT primary outcomes (AD dementia incidence, cognitive decline) showed no longterm benefit of either NSAID (29).

\section{Delayed-washout evidence of disease modification?}

An unintended aspect of the termination of the ADAPT treatments was its realization of a "delayed washout" design of the sort typified by the DATATOP trial of deprenyl for Parkinson's disease (30), and discussed in Paul Leber's classic paper on differentiation of symptomatic vs. disease-modifying treatments for AD (31). By the time the ADAPT treatments had been stopped, cumulative hazard analyses of ADAPT suggested that the incidence of dementia in the placeboassigned group had "caught up" with the (elevated) incidence among those assigned to the NSAID treatments (27). Only after this point, however, did naproxenassigned subjects appear to show reduced incidence of dementia for several years. Likewise, a contrast in CSF biomarkers favoring naproxen-treated subjects was observed in the years after termination of treatments (27). These observations well after the (unplanned) interruption of the ADAPT treatments suggest that a delayed-washout design may be used for other tests of interventions in the pre-symptomatic space.

\section{Development of a new Centre for Studies on Prevention of AD}

In 2006 an influential paper by Leon Thal emphasized the potential importance of safety concerns and advocated prevention approaches that relied on behavioural or "lifestyle" interventions (32). Several prevention trials of this sort have now been undertaken, some with encouraging results (33-35). Concerns remain, however about the feasibility of persuading large numbers of people to change their behaviour in accord with these trials' intervention strategies, and a search for pharmaco-prevention strategies remains important (consider, by analogy, the success of medical vs. behavioural treatments for Type II diabetes, hyperlipidemias, or hypertension).

By contrast, the potential safety concerns of drug treatments tend to be problematic for long-term prevention trials, where there is no proven benefit to offset risks that become apparent in "real time" (36). More generally, it is difficult to identify the most promising candidate pharmaco-preventive interventions. In late 2010, therefore, we began an ambitious new initiative aimed at facilitating the discovery of safe and promising pharmacologic interventions that appeared to slow the progression of pre-symptomatic AD. We began with several premises: 
1. To prevent AD symptoms, one must slow (or arrest) the progress of the disease in its pre-symptomatic stage. The latter may even include times when functional or chemical change is demonstrable before there is evident structural pathology (e.g., fibrillar $A \beta$ deposits).

2. Working in this pre-symptomatic space, one cannot rely on occurrence or progression of typical AD symptoms (excepting, at least in theory, cognitive decline over time).

3. By elimination, one must therefore concentrate on biomarkers of $\mathrm{AD}$ progression.

4. We did not know which biomarkers would best serve this purpose, but it seemed likely that (given the heterogeneity in $\mathrm{AD}$ progression) multiple markers would be advantageous.

5. Such study of multiple markers was certain to create a challenge in finding methods of analysis to measure treatment effects on the pathogenesis of the disease. We thought it logical, nonetheless, to begin our inquiries with several markers most robustly associated with the later, symptomatic stages of the disease, realizing that at some later point we would need to validate the chosen markers as being indicative of the pre-symptomatic disease process.

6. For practical reasons, we recognized that we would need sufficient precision in the measurement of change in the marker indicators over a relatively brief interval (we set a goal of two years for robust detection of such change).

7. Our research subjects needed to be at substantially elevated risk of $\mathrm{AD}$ dementia. Ideally, a substantial majority of them should be at some stage of presymptomatic AD.

8. We preferred, however, to avoid restriction of participation to "special populations", because we wanted results that could plausibly apply to typical populations in general (or at least to a substantial element of such populations). For this reason, we did not wish to resort to APOE genotyping as a means of "enriching" our sample for persons with presymptomatic $\mathrm{AD}$.

9. Within limits of available resources, we wished to measure as many biomarkers as possible at regular intervals (in the parlance of the UK Dementia Platform, "Deep and Frequent Phenotyping"). Because we wanted ideally to find markers that changed over two years, we planned to examine participants annually.

10. To the extent possible, our hope was to "let the data tell us" which markers show most readily detectable change.

Complementing these ideas, we believed it would be important to assess whether the encouraging biomarker results with naproxen from ADAPT could be corroborated. We therefore elected to undertake a biomarker-endpoint trial of naproxen as a practical way to test the feasibility of this approach.

\section{The PREVENT-AD Cohort}

Thus, in late 2011, our Centre began to assemble a cohort of participants for PRe-symptomatic EValuation of Experimental or Novel Treatments for AD (PREVENTAD). Participants in this Cohort have either a parental history of Alzheimer-type dementia or multiple siblings affected by the disease. Other entry criteria were designed to assure the possibility of long-term followup studies using multiple biomarker endpoints, and

Table 1. Selected demographics of the observational PREVENT-AD Cohort, the nested INTERPAD trial, and its panel of serial CSF donors

\begin{tabular}{|c|c|c|c|}
\hline Variable mean (s.d.) & $\begin{array}{c}\text { non-trial COHORT } \\
\mathbf{n}=\mathbf{1 3 1}\end{array}$ & $\begin{array}{l}\text { INTREPAD } \\
\mathrm{m}-\mathrm{ITT} \mathrm{n}=160\end{array}$ & $\begin{array}{l}\text { INTREPAD m-ITT } \\
\text { LP volunteers } \mathrm{n}-94\end{array}$ \\
\hline Age of participants (s.d.) & $63(3)$ & $63(6)$ & $63(6)$ \\
\hline $\begin{array}{l}\text { age of parental onset of AD } \\
\text { (s.d.) } \\
\text { (youngest if }>1 \text { affected) }\end{array}$ & $72(3)$ & $73(8)$ & $73(8)$ \\
\hline $\begin{array}{l}\text { Number of years to parental } \\
\text { onset of AD }\end{array}$ & $\begin{array}{c}-9(8) \\
\text { Range }-25-+23\end{array}$ & $\begin{array}{c}-10(8) \\
\text { Range }-28-+23\end{array}$ & $\begin{array}{c}-11(8) \\
\text { Range }-28-+23\end{array}$ \\
\hline Gender & $30 \%$ male & $25 \%$ male & $31 \%$ male \\
\hline Years of education & $16(3)$ & $15(3)$ & $15(3)$ \\
\hline APOE \& 4 carriers $(\%)$ & $\begin{array}{c}(\mathrm{n}=108 \text { analyzed } \\
37(34 \%) \text { carriers } \\
4 \text { homozygotes }\end{array}$ & $\begin{array}{c}55(34 \%) \text { carriers } \\
3 \text { homozygotes }\end{array}$ & $\begin{array}{c}32 \text { carriers }(35 \%) \\
0 \text { homozygotes }\end{array}$ \\
\hline MoCA score (out of 30) & $28(1.5)$ & $28(1.6)$ & $28(1.5)$ \\
\hline
\end{tabular}

mITT $=$ modified Intend-to-Treat (subjects with at least one follow-up data point) 
Table 2. Multiple metrics for analysis as indicators of AD pathogenesis

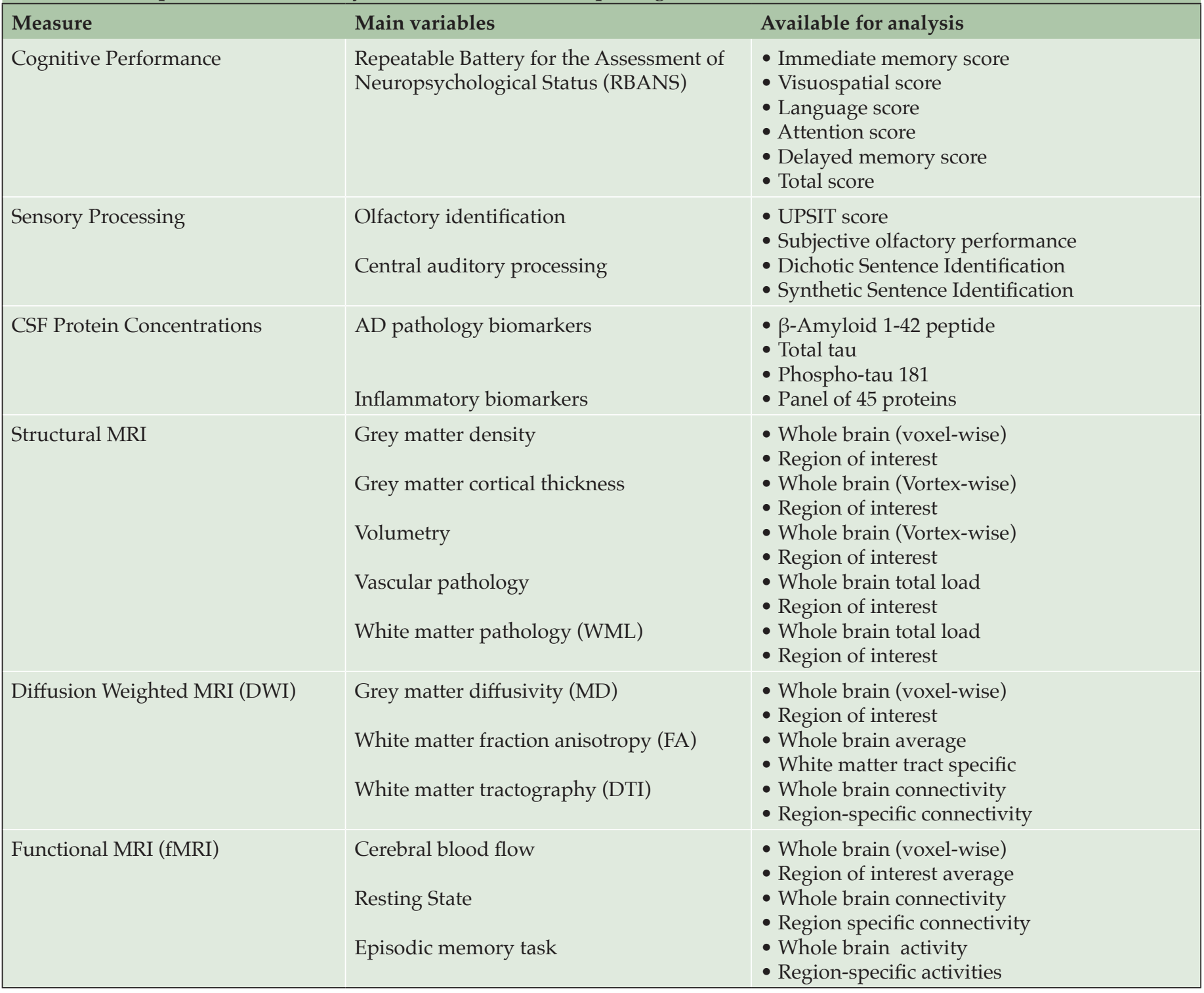

the design called for annual follow-up examinations using cognitive and multiple-biomarker measures to indicate apparent progression of pre-symptomatic AD. A subset of this longitudinal cohort study were enrolled in a two-year biomarker-endpoint trial of the traditional (dual-inhibitor) NSAID naproxen sodium 220 mg tablets (available without prescription in Canada). After receiving approval from Health Canada and our institutional ethics committee, we began enrollment of a target of 200 persons for this randomized, placebocontrolled, double-masked trial, which we named INTREPAD (Impact of Naproxen TREatment in Presymptomatic Alzheimer's Disease; NCT 02702817). Naproxen sodium $220 \mathrm{mg}$ tablets and matching placebo for this double-blind, randomized trial were generously provided by Pharmascience, Inc., a prominent Canadian manufacturer of generic pharmaceuticals.

All participants in both the PREVENT-AD Cohort and its sister INTREPAD trial provided written informed consent prior to enrollment. Characteristics of these participants are shown in Table 1 . Table 2 enumerates the biomarker endpoints under investigation by these efforts. At each point of evaluation INTREPAD participants (but not other members of the non-trial PREVENT-AD cohort) were asked to donate CSF via LP, and over half of them agreed to a series of four CSF donations over a two-year interval (Figure 1).

\section{Value of simultaneous studies among INTREPAD and non-trial PREVENT-AD participants}

As these studies were designed, we were uncertain which among the multiple markers to be followed in INTREPAD would best serve as endpoints for the trial. 
We therefore chose to "let the data (from longitudinal observations in the non-trial PREVENT-AD Cohort) tell us" which markers or combinations of markers would most likely indicate the progress of pre-symptomatic disease. While remaining committed to the principle that data collection methods must be fully specified before the trial began, we nonetheless opted to suspend declaration of the trial's outcomes pending observation of substantial longitudinal data from the non-trial cohort. It was hoped that the latter could reveal in "real time" which markers (excluding CSF analytes that were not available) would best serve as outcomes for the doublemasked INTREPAD trial. Analysis of these data then permitted declaration of outcomes (see below) before beginning analyses of trial treatment effects.

\section{Figure 1. Completed Lumbar Punctures}

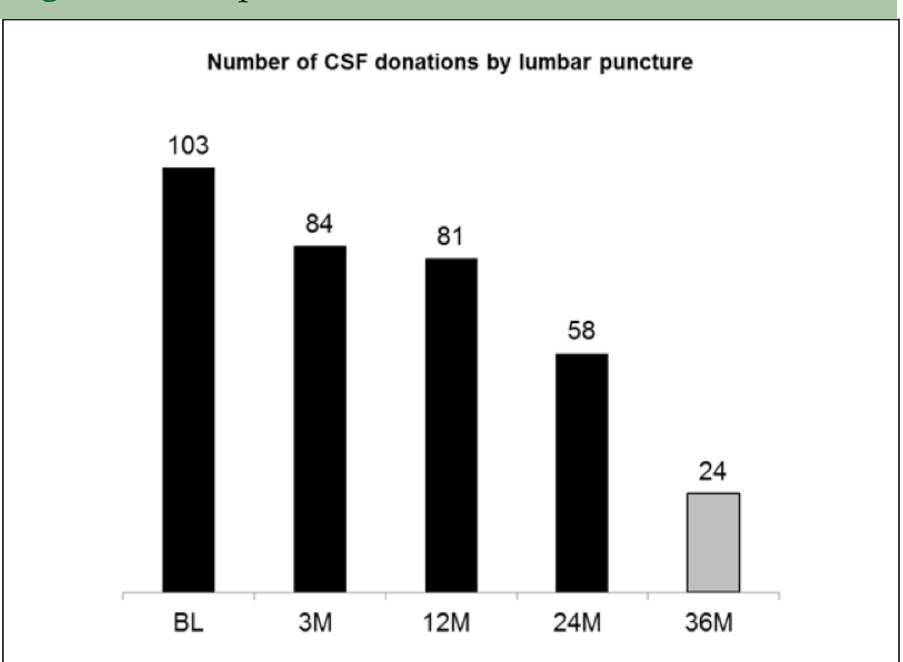

Legend: INTREPAD participants were asked to volunteer for a series of lumbar punctures at Baseline (BL) and at specified follow-up intervals of 3, 12, and 24 months when they return for examination. Of the 103 who volunteered for LPs, 94 stayed on protocol for at least three months and, as members of the m-ITT pool, were asked to continue the series. Over half the INTREPAD m-ITT participants have now completed their 24-month follow-up visit and are off study drug. Of these, 24 have returned for annual assessment in the post-treatment "delayed washout" phase of the trial. About 80 persons are expected eventually to complete follow-up by spring 2017 .

\section{Consideration of novel marker systems}

We have chosen to pursue several CSF and either structural or functional imaging variables for investigation as potential markers of pre-symptomatic disease progression. Table 2 shows a number of traditional and non-traditional marker systems, including two sensori-neural abilities as candidate pre-symptomatic AD biomarkers. Abilities in olfactory identification are impaired in Alzheimer's dementia and MCI (37, 38) and prodromal $\mathrm{AD}$ (39), and may even show some relationship to treatment response to cholinesterase inhibiting drugs (40). We therefore added the University of Pennsylvania Smell Identification Test (UPSIT) to our annual assessment battery (41) finding substantial correlations of olfactory identification with age, and with several other biomarker endpoint assessments reported at the 2015 and 2016 AAIC meetings. As well, we explored the utility of two tests of central auditory processing (CAP) as possible indicators of pre-clinical disease progression, relying especially on a powerful observational study by Gates et al. (42), that identified subjects with impaired CAP among cognitively normal members of the Adult Changes in Thought (ACT) study directed by Eric Larson. Over four years of follow-up, ACT participants with impaired CAP had shown up to nine-fold increases in incidence of AD dementia.

\section{A principal outcome conjoining two main outcomes}

As an exception to the "let the data tell us" approach, we committed a priori to consider global cognitive ability as an important trial endpoint. We reasoned that, even without a demonstrable effect on biologically oriented measures, a treatment effect of diminished cognitive decline would relate intuitively to prevention of cognitive disorder, and would anyway be valuable in its own right. For such a cognitive endpoint, we chose total score on the 30-40 minute Repeatable Battery for Assessment of Neuropsychological Status (43). This battery is available in four equivalent versions in Canadian French and is suited to the study of cognitive abilities in persons without dementia (for example, having good sensitivity in separating MCI from normal cognition, it is now being used as an entry criterion in three pharmaceutical trials with MCI endpoints). The RBANS comprises 12 individual tests that generate scores on five scales. The global and all scale scores have psychometric properties modeled on the WAIS (typical median score 100; s.d. = 15 points).

For the remaining (non-cognitive) main outcome we needed a summary variable that could provide a digest of the multimodal biomarker and sensori-neural marker data available to indicate the degree of pre-symptomatic disease progression. The idea of a single such summary score grew from our observation of the inter-relatedness, in the non-trial PREVENT-AD cohort, of many of the markers shown in Table 2 (data not shown). Most of these correlated strongly with age, but the latter correlation was typically vitiated when other presumed AD markers were included in multivariable models. By contrast, strong apparent correlations among these biomarkers suggested that many of them were common effects of an underlying "driver," and we hypothesized that the latter might in fact be the progression of pre-symptomatic $\mathrm{AD}$ pathogenesis. We therefore developed a latent variable modeling method that yields a single 'Alzheimer Progression Score.' The development and validation of this APS is described in an accompanying paper (45). Briefly, the score is estimated using item response theory to analyze the data on multiple individual disease markers, including CSF analytes when available.

How, then, to consider the two main outcomes 
together for examination of treatment effects? If we accepted either outcome as being a sufficient test of the null hypothesis (of no treatment effect), this would require correction for multiple comparisons with resulting loss of statistical power. By contrast, requiring that both achieve some critical p-value (alpha) would be too stringent (again, with risk of type II error). If the two main outcomes were independent (they are not), one might avoid this conundrum by calculating their joint probability using simple multiplication (e.g., two observed p-values of 0.1 would result in a combined $p=0.01$ ). Instead, we have adapted a method (to be discussed in a forthcoming report) that considers the joint probabilities of both main outcomes while taking into account the correlation between them. We suggest that this approach should achieve the "best of both worlds" as it considers cognition separately from biological markers but uses information from both to estimate disease progress.

\section{Looking forward ...}

It will be about six months before the last-enrolled participant in INTREPAD will undergo his/her final assessment on-treatment, after which we shall unmask treatment assignment. Should the trial show a treatment effect of naproxen, we shall (of course) be strongly motivated to continue to study antiinflammatory treatments as a route to the prevention of AD. We plan also to extend the observations of the trial cohort off-treatment for another two years, with annual assessments, to ascertain whether any observed treatment effects (whatever their level) are sustained, as would be expected if naproxen did indeed have a diseasemodifying influence on the pathogenesis of pre-clinical $\mathrm{AD}(31)$.

In analogous fashion, we have begun a new program of evaluation of the potential as an $\mathrm{AD}$ preventive of the retired cholesterol-lowering drug probucol as a putative stimulator of increased availability of the apoE protein. And, in separate studies, we are using high-throughput technology from both Millipore and MesoScale Discovery to analyze up to 45 different markers of inflammation and immune activity in the CSF of those INTREPAD participants who have undergone multiple LPs (44).

We suggest, however, that the most important result of our work will not be its answer regarding the apparent efficacy of naproxen (or probucol) as inhibitors of preclinical disease progression. Whatever the results of these trials, their successful completion should provide a demonstration of the feasibility of the described method of biomarker-endpoint trials in high-risk individuals for economical testing of the likely success of any individual candidate preventive agent. To date, for example, the work on INTREPAD has cost approximately $\$ 3.0$ million CAD (US\$ 2.3 million), and we expect to complete it with other funds totalling no more than \$2.0 million.
Considering the many years and scores of millions of dollars required to undertake a Phase III prevention trial using incident illness (Alzheimer's dementia or even $\mathrm{MCI} / \mathrm{AD}$ ) as an endpoint, we suggest that the described method may provide important preliminary data at much lower cost to justify this sort of investment. Even without confirmation of a naproxen effect on AD pathogenesis, we suggest that the data from INTREPAD, as well as the remainder of the PREVENT-AD Cohort will prove valuable for analyses of the signs of $A D$ pathogenesis in high-risk older persons. Thus the work may provide valuable information along many dimensions. We trust that the resulting data and results of their analyses may provide helpful examples more broadly for the field of AD prevention.

Funding: Funded by generous support from McGill University, the government of Canada, an unrestricted gift from Pfizer Canada, the Canada Fund for Innovation, the Douglas Hospital Research Centre, the Levesque Foundation. Naproxen sodium $220 \mathrm{mg}$ tablets and matching placebo and probucol $300 \mathrm{mg}$ scored tablets and their placebo have been generously provided by Pharmascience Inc. The sponsors had no role in the design and conduct of the studies; in the collection, analysis, and interpretation of data; in the preparation of the manuscript; or in the review or approval of the manuscript.

Conflicts: The authors declare that they have no conflicts of interest with this work

Acknowledgements: The entire PREVENT-AD Research Group, past and present, is listed at https://preventad.loris.ca/team_2016_09_16.pdf. We are deeply grateful for the tireless generosity and commitment of the participants in our work, and for helpful comments and the personal commitment of Prof. Alan Evans and Mr. Samir Das of the McConnell Brain Imaging Center, Montreal Neurological Institute. We also thank Prof. Remi Quirion, former Dean Richard Levin, and Mr. Jacques Hendlisz, without whose encouragement and commitment this work would not have been undertaken, and Drs. Alain Gratton, Mimi Israel, and Gustavo Turecki for their key efforts to help along the way.

Ethical standards: All research was conducted under the principles of the World Medical Association Declaration of Helsinki, and all procedures were approved by the IRB of the McGill University Faculty of Medicine. Experimental administration of both naproxen sodium and probucol for this work was approved by Health Canada.

\section{References}

1. Breitner, J.C.S. and M.F. Folstein, Familial Alzheimer dementia: a prevalent disorder with specific clinical features. Psychological Medicine, 1984. 14: p. 63-80

2. Breitner, J.C.S., E.A. Murphy, and M.F. Folstein, Familial aggregation in Alzheimer dementia II: clinical genetic implications of age-dependent onset. Journal of Psychiatric Research, 1986. 20: p. 45-55

3. Breitner, J.C.S., M.F. Folstein, and E.A. Murphy, Familial aggregation in Alzheimer dementia I: a model for the age-dependent expression of an autosomal dominant gene. Journal of Psychiatric Research, 1986. 20(1): p. 31-43

4. Farrer, L.A., D.M. O'Sullivan, L.A. Cupples, J.H. Growdon, and R.H. Myers, Assessment of genetic risk for Alzheimer's disease among first-degree relatives. Annals of Neurology, 1989. 25: p. 485-493

5. Breitner, J.C.S., Clinical genetics and genetic counseling in Alzheimer's disease. Annals of Internal Medicine, 1991. 115: p. 601-606

6. Khachaturian, Z., The five-five, ten-ten plan for Alzheimer's disease Neurobiol Aging, 1992. 13(2): p. 197-8; discussion 199. PMID:1522938

7. Brookmeyer, R., S. Gray, and S. Kawas, Projections of Alzheimer's disease in the United States and the public health impact of delaying disease onset. Am J Public Health, 1998. 88(9): p. 1337-1342

8. Strittmatter, W.J., K.H. Weisgraber, D.Y. Huang, L.M. Dong, G.S. Salvesen, M. Pericak-Vance, et al., Binding of human apolipoprotein E to synthetic amyloid B peptide: isoform-specific effects and implications for late-onset Alzheimer disease. Proceedings of the National Academy of Sciences of the United States of America, 1993. 90: p. 8098-8102

9. Poirier, J., J. Davignon, D. Bouthillier, S. Kogan, P. Bertrand, and S. Gauthier, Apolipoprotein E polymorphism and Alzheimer's disease. Lancet, 1993. 342 p. 697-699

10. Corder, E.H., A.M. Saunders, W.J. Strittmatter, D.E. Schmechel, P.C. Gaskell, 
G.W. Small, et al., Gene dose of apolipoprotein E type 4 allele and the risk of Alzheimer's disease in late onset families. Science, 1993. 261: p. 921-923

11. Myers, R.H., E.J. Schaefer, P.W.F. Wilson, R. D'Agostino, J.M. Ordovas, A. Espino, et al., Apolipoprotein E e4 association with dementia in a populationbased study: the Framingham Study. Neurology, 1996. 46: p. 673-677

12. Breitner, J.C.S., B.W. Wyse, J.C. Anthony, K.A. Welsh-Bohmer, D.C. Steffens, M.C. Norton, et al., APOEe4 count predicts age when prevalence of Alzheimer's disease increases--then declines. The Cache County Study. Neurology, 1999. 53: p. 321 - 331

13. Miech, R., J. Breitner, P.P. Zandi, A. Khachaturian, J. Anthony, and L. Mayer, Incidence of AD may decline in the early 90 s for men, later for women: The Cache County Study. Neurology, 2002. 58(2): p. 209-218

14. Meyer, M.R., J.T. Tschanz, M.C. Norton, K.A. Welsh-Bohmer, D.C. Steffens, B.W. Wyse, et al., APOE genotype predicts when--not whether--one is predisposed to develop Alzheimer disease. Nat Genet, 1998. 19(4): p. 321-2. PMID:9697689

15. Breitner, J.C.S. and K.A. Welsh, Genes and recent developments in the epidemiology of Alzheimer's disease and related dementia. Epidemiologic Reviews, 1995. 17: p. 39-47

16. Hardy, J.A. and G.A. Higgins, Alzheimer's disease: the amyloid cascade hypothesis. Science, 1992. 256: p. 184-185

17. Kosik, K.S., Tau protein and Alzheimer's disease. Current Opinion in Cell Biology, 1990. 2: p. 101-104

18. Reiman, E.M., J.B. Langbaum, A.S. Fleisher, R.J. Caselli, K. Chen, N Ayutyanont, et al., Alzheimer's Prevention Initiative: a plan to accelerate the evaluation of presymptomatic treatments. J Alzheimers Dis, 2011. 26 Suppl 3: p. 321-9. PMID:21971471

19. Mills, S.M., J. Mallmann, A.M. Santacruz, A. Fuqua, M. Carril, P.S. Aisen, et al., Preclinical trials in autosomal dominant AD: implementation of the DIAN-TU trial. Rev Neurol (Paris), 2013. 169(10): p. 737-43. PMID:24016464

20. Wischik, C.M., R.T. Staff, D.J. Wischik, P. Bentham, A.D. Murray, J.M. Storey, et al., Tau aggregation inhibitor therapy: an exploratory phase 2 study in mild or moderate Alzheimer's disease. J Alzheimers Dis, 2015. 44(2): p. 70520. PMID:25550228

21. Fagan, T. In First Phase 3 Trial, the Tau Drug LMTM Did Not Work. Period. 2016; Available from: http:/ / www.alzforum.org/news/conferencecoverage/alzheimers-association-international-conference-2016.

22. Mawuenyega, K.G., W. Sigurdson, V. Ovod, L. Munsell, T. Kasten, J.C. Morris, et al., Decreased clearance of CNS beta-amyloid in Alzheimer's disease. Science, 2010. 330(6012): p. 1774. PMID:21148344

23. Iturria-Medina, Y., R.C. Sotero, P.J. Toussaint, A.C. Evans, and I. Alzheimer's Disease Neuroimaging, Epidemic spreading model to characterize misfolded proteins propagation in aging and associated neurodegenerative disorders. PLoS Comput Biol, 2014. 10(11): p. e1003956. PMID:25412207

24. Heneka, M.T., M.J. Carson, J. El Khoury, G.E. Landreth, F. Brosseron, D.L. Feinstein, et al., Neuroinflammation in Alzheimer's disease. Lancet Neurol, 2015. 14(4): p. 388-405. PMID:25792098

25. Martin, B., C.L. Meinert, and J. Breitner, Double placebo design in a prevention trial for Alzheimer's disease. Control Clin Trials, 2002. 23(1): p 93-9.

26. ADAPT Research Group, C.G. Lyketsos, J.C. Breitner, R.C. Green, B.K. Martin, C. Meinert, et al., Naproxen and celecoxib do not prevent AD in early results from a randomized controlled trial. Neurology, 2007. 68(21): p. 1800-8. PMID:17460158

27. Breitner, J.C., L.D. Baker, T.J. Montine, C.L. Meinert, C.G. Lyketsos, K.H. Ashe, et al., Extended results of the Alzheimer's disease anti-inflammatory prevention trial. Alzheimers Dement, 2011. 7(4): p. 402-11. PMID:21784351

28. Leoutsakos, J.M., B.O. Muthen, J.C. Breitner, C.G. Lyketsos, and A.R. Team, Effects of non-steroidal anti-inflammatory drug treatments on cognitive decline vary by phase of pre-clinical Alzheimer disease: findings from the randomized controlled Alzheimer's Disease Anti-inflammatory Prevention
Trial. Int J Geriatr Psychiatry, 2012. 27(4): p. 364-74. PMID:21560159

29. Alzheimer's Disease Anti-inflammatory Prevention Trial Research, G. Results of a follow-up study to the randomized Alzheimer's Disease Antiinflammatory Prevention Trial (ADAPT). Alzheimers Dement, 2013. 9(6): p. 714-23. PMID:23562431

30. LeWitt, P.A., Deprenyl's effect at slowing progression of parkinsonian disability: the DATATOP study. Acta Neurologica Scandinavica, 1991. 84(S136): p. 79-86

31. Leber, P., Observations and suggestions on antidementia drug development Alzheimer Dis Assoc Disord, 1996. 10 Suppl 1: p. 31-5. PMID:8876787

32. Thal, L.J., Prevention of Alzheimer disease. Alzheimer Dis Assoc Disord, 2006. 20(3 Suppl 2): p. S97-9. PMID:16917204

33. Kivipelto, M. FINGER - A multidomain two-year randomized trial to prevent cognitive decline. in Clinical Trials on Alzheimer's Disease. 2015. Spain: JPAD.

34. Dufouil, C. Impact of a multi-domain intervention on brain atrophy rate: the MAPT MRI ancillary study. in Clinical Trials on Alzheimer's Disease. 2015 Spain: JPAD.

35. Baker, L. Aerobic exercise reduces phosphorylated tau protein in cerebrospinal fluid in older adults with mild cognitive impairment in Clinical Trials on Alzheimer's Disease. 2015. Spain: JPAD.

36. Meinert, C.L. and J.C. Breitner, Chronic disease long-term drug prevention trials: lessons from the Alzheimer's Disease Anti-inflammatory Prevention Trial (ADAPT). Alzheimers Dement, 2008. 4(1 Suppl 1): p. S7-S14. PMID:18632005

37. Sun, G.H., C.A. Raji, M.P. Maceachern, and J.F. Burke, Olfactory identification testing as a predictor of the development of Alzheimer's dementia: a systematic review. Laryngoscope, 2012. 122(7): p. 1455-62. PMID:22552846

38. Rahayel, S., J. Frasnelli, and S. Joubert, The effect of Alzheimer's disease and Parkinson's disease on olfaction: a meta-analysis. Behav Brain Res, 2012. 231(1): p. 60-74. PMID:22414849

39. Roberts, R.O., T.J. Christianson, W.K. Kremers, M.M. Mielke, M.M Machulda, M. Vassilaki, et al., Association Between Olfactory Dysfunction and Amnestic Mild Cognitive Impairment and Alzheimer Disease Dementia. JAMA Neurol, 2015: p. 1-9. PMID:26569387

40. Velayudhan, L. and S. Lovestone, Smell identification test as a treatment response marker in patients with Alzheimer disease receiving donepezil. J Clin Psychopharmacol, 2009. 29(4): p. 387-90. PMID:19593181

41. Doty, R.L., P. Shaman, and M. Dann, Development of the University of Pennsylvania Smell Identification Test: a standardized microencapsulated test of olfactory function. Physiol Behav, 1984. 32(3): p. 489-502. PMID:6463130

42. Gates, G.A., L.E. Gibbons, S.M. McCurry, P.K. Crane, M.P. Feeney, and E.B Larson, Executive dysfunction and presbycusis in older persons with and without memory loss and dementia. Cogn Behav Neurol, 2010. 23(4): p. 21823. PMID:21150347

43. Randolph, C., M.C. Tierney, E. Mohr, and T.N. Chase, The Repeatable Battery for the Assessment of Neuropsychological Status (RBANS): preliminary clinical validity. J Clin Exp Neuropsychol, 1998. 20(3): p. 310-9. PMID:9845158

44. Breitner, J.S.C., J. Poirier, T. Town, P. Rosa-Neto, J. Tremblay-Mercier, T.M. Weitz, et al., CSF Markers of Inflammation and Alzheimer's Disease Pathogenesis in the Cognitively Intact Prevent-AD Cohort in Alzheimer's Association International Conference 2016: Toronto.

45. Leoutsakos J.-M., Gross A.L., Jones R.N., Albert M.S., Breitner J.C. 'Alzheimer's Progression Score': Development of a Biomarker Summary Outcome for AD Prevention Trials. J Prev Alz Dis. in press 